\section{KNOWLEDGE AND UNDERSTANDING OF HEALTHCARE ETHICS: A SURVEY OF PALLIATIVE CARE TEAMS AT THE PRINCE AND PRINCESS OF WALES HOSPICE AND THE QUEEN ELIZABETH UNIVERSITY HOSPITAL IN GLASGOW}

${ }^{1}$ Marisa Devanney, ${ }^{2}$ Carolyn Datta. ${ }^{1}$ University of Glasgow; ${ }^{2}$ Prince and Princess of Wales Hospice

10.1136/bmjspcare-2019-ASP.52

Introduction Ethics is integral to palliative care, making staff knowledge of healthcare ethics critical. A survey was distributed to palliative care teams at the Prince and Princess of Wales Hospice and Queen Elizabeth University Hospital in Glasgow to assess staff self-reported knowledge of ethics and ethical issues encountered.

Aims

- To assess staff knowledge of ethics and confidence in applying knowledge;

- To determine the incidence of ethical issues in palliative care;

- To gain insight into the types of ethical issues staff encounter in their work and how they currently deal with these;

- To assess the desire for further staff training in ethics.

Methods The survey was distributed to healthcare assistants, nurses, doctors, allied healthcare professionals and family support services at a hospice and hospital. Data was analysed using Microsoft Excel.

Results

- 35 out of 75 surveys were completed; 51\% nurses, 23\% healthcare assistants, $17 \%$ doctors, $3 \%$ allied healthcare professionals, and 6\% family support services;

- $69 \%$ of participants had training in healthcare ethics;

- Participants demonstrated a high knowledge of the four main principles of ethics;

- There was ambiguity as to what constitutes an ethical issue;

- $86 \%$ had encountered at least one ethical issue at work;

- Most commonly reported issue was a patient who stopped eating, cited by $44 \%$ of hospice participants;

- $50 \%$ of hospital staff raised concerns about medical interventions at end of life;

- Majority of respondents felt able to deal with these ethical issues;

- Participants often turned to colleagues for support, benefiting from discussing ethical issues;

- $77 \%$ desired ethical training; a course was the most popular method.

Conclusion

- Staff knowledge of ethics appears solid;

- Self-reported staff confidence measures on applying ethical knowledge was high;

- There is a high incidence of ethical issues in palliative care;

- Common ethical issues reported were patients stopping eating and issues surrounding medical interventions;

- Majority of staff were desirous of additional ethical training.

\section{IMPROVING INPATIENT ANTI-EMETIC PRESCRIBING AT THE ROYAL MARSDEN HOSPITAL}

Stephanie Flynn, Daniel Westaby. The Royal Marsden Hospital
Background Whilst working on the adult inpatient wards at The Royal Marsden Hospital (RMH), it was noted that antiemetics were frequently prescribed incorrectly particularly in combination (e.g. metoclopramide with domperidone, or metoclopramide with cyclizine). We felt a quality improvement project that included a simple intervention involving a teaching session to junior doctors on anti-emetic prescribing could improve knowledge and practice.

Methods Data was collected from inpatient medication charts, both before and after the intervention. We aimed to collect data from 25-50 charts with at least one anti-emetic prescription. All anti-emetic prescriptions were recorded along with any prescribing errors (including incorrect combination, incorrect dose/frequency/max dose/route).

A teaching session was delivered to all RMH SHOs highlighting anti-emetic interactions/cautions and safe prescribing practice. SHOs were also asked to complete a questionnaire before and after the teaching session to assess confidence in anti-emetic prescribing.

Results Drug charts were analysed from 37 patients between $13 / 03 / 18$ and 10/04/18. 5 prescribing errors were found (13.5\%). 37 new drug charts were analysed 1 week after the intervention. 0 prescribing errors were found (0\%). SHO questionnaires were completed with an average confidence level of 3.1 (out of 5) before the intervention and 4.3 after. Conclusions The results show a reduction in prescribing errors and an improvement in prescribing confidence post-intervention. This shows that simple teaching interventions can improve prescribing safety and confidence.

We plan to implement a similar teaching session at induction for each new cohort of junior doctors to ensure these changes are lasting. SHOs rotating to palliative care/oncology placements are often not familiar with prescribing anti-emetic combinations as they have usually come from other medical specialities where their use is less common. This small project has shown how targeted teaching interventions can improve anti-emetic prescribing practice and patient care, and could be easily replicated at other centres.

\section{End of Life Care | Posters $31-67$}

\section{LEARNING FROM HOSPICE EXPERIENCES OF WITHDRAWING CONTINUOUS ARTIFICIAL NUTRITION AND HYDRATION IN PATIENTS WITH PROLONGED DISORDERS OF CONSCIOUSNESS}

Heledd Lewis, Madeleine Carr, Siwan Seaman. Marie Curie Hospice Cardiff and the Vale

\subsection{6/bmjspcare-2019-ASP.54}

Background Following The Supreme Court judgement it is no longer mandatory to involve the court to make best interest decisions to withdraw clinically assisted nutrition and hydration $(\mathrm{CANH})$ in patients with prolonged disorders of consciousness (PDOC). We anticipate a higher number of patients being admitted to the hospice setting for end of life care for this indication.

Method Reviewed case notes of patients, with a diagnosis of PDOC, admitted to our hospice for discontinuation of $\mathrm{CANH}$ and analysed the content of interviews with multi-disciplinary health care professionals who had been involved in managing and caring for these patients. 